\title{
A GAP THEOREM FOR ENDS OF COMPLETE MANIFOLDS
}

\author{
MINGLIANG CAI, TOBIAS HOLCK COLDING, AND DAGANG YANG
}

(Communicated by Peter Li)

\begin{abstract}
Let $\left(M^{n}, o\right)$ be a pointed open complete manifold with Ricci curvature bounded from below by $-(n-1) \Lambda^{2}$ (for $\Lambda \geq 0$ ) and nonnegative outside the ball $B(o, a)$. It has recently been shown that there is an upper bound for the number of ends of such a manifold which depends only on $\Lambda a$ and the dimension $n$ of the manifold $M^{n}$. We will give a gap theorem in this paper which shows that there exists an $\varepsilon=\varepsilon(n)>0$ such that $M^{n}$ has at most two ends if $\Lambda a \leq \varepsilon(n)$. We also give examples to show that, in dimension $n \geq 4$, such manifolds in general do not carry any complete metric with nonnegative Ricci Curvature for any $\Lambda a>0$.
\end{abstract}

\section{INTRODUCTION}

The Cheeger-Gromoll splitting theorem states that in a complete manifold of nonnegative Ricci curvature, a line splits off isometrically, i.e., any nonnegatively Ricci curved $M^{n}$ is isometric to a Riemannian product $N^{k} \times \mathbf{R}^{n-k}$, where $N$ does not contain a line (cf. [CG]). In particular, such a manifold has at most two ends. Recently, the first-named author and independently $\mathrm{Li}$ and Tam have shown that a complete manifold with nonnegative Ricci curvature outside a compact set has at most finitely many ends [C, LT]. At about the same time, Liu has also given a proof of the same theorem with an additional condition that there is a lower bound on sectional curvature [L], which was removed shortly after the appearance of [C]. In this paper, we consider manifolds with nonnegative Ricci curvature outside a compact set and prove the following gap theorem.

Theorem. Given $n>0$, there exists an $\varepsilon=\varepsilon(n)>0$ such that for all pointed open complete manifolds $\left(M^{n}, o\right)$ with Ricci curvature bounded from below by $-(n-1) \Lambda^{2}$ (for $\left.\Lambda \geq 0\right)$ and nonnegative outside the ball $B(o, a)$, if $\Lambda a \leq \varepsilon(n)$, then $M^{n}$ has at most two ends.

A natural question one would like to ask is whether this theorem can be improved so that $M^{n}$ must carry a complete metric with nonnegative Ricci curvature. Indeed, it is easy to see by volume comparison that the answer to the above question is affirmative in dimension 2 since the Euler number of such

Received by the editors April 6, 1993.

1991 Mathematics Subject Classification. Primary 53C20.

The third author was partially supported by National Science Foundation grant DMS 90-03524. 
a 2-dimensional complete manifold is an upper bound for the total curvature integral. However, such a gap theorem is the best one can have in dimensions higher than 3 as illustrated by the following examples.

For any $\varepsilon>0$, by gluing two sharp cones together at the singular point, it is easy to construct a complete metric on $R \times S^{n-2}, n \geq 4$, with Ricci curvature bounded from below by $-\varepsilon$ and with nonnegative sectional curvature away from a metric ball of radius 1 . By applying the metric surgery techniques as in [SY] to the manifold $S^{1} \times R \times S^{n-2}$, one obtains an $n$-dimensional complete manifold $M$ of infinite homotopy type with exactly two ends and with Ricci curvature bounded from below by $-\varepsilon$ and with nonnegative Ricci curvature outside a metric ball of radius $1 . M$ certainly cannot carry any complete metric with nonnegative Ricci curvature since the Cheeger-Gromoll splitting theorem implies that a nonnegatively Ricci curved manifold with exactly two ends must split isometrically into the product of $R$ with a closed manifold and therefore has finite homotopy type.

The above examples are not valid in dimension 3 since the kind of metric surgery lemmas are not available. Therefore, the following problem is of particular interest:

Does there exist an $\varepsilon>0$ such that if $(M, o)$ is a pointed noncompact complete 3-dimensional manifold with Ricci curvature bounded from below by $-\varepsilon$ and nonnegative outside the unit metric ball $B(o, 1)$, then $M$ carries a complete metric with nonnegative Ricci curvature?

\section{Proof of the theorem}

There are various (but equivalent) definitions of an end of a manifold. For the sake of our argument, we use the following (compare with [A]).

Definition 2.1. Two rays $\gamma_{1}$ and $\gamma_{2}$ starting at the base point $o$ are called cofinal, if for any $r \geq 0$ and all $t \geq r, \gamma_{1}(t)$ and $\gamma_{2}(t)$ lie in the same component of $M-B(o, r)$. An equivalence class of cofinal rays is called an end of $M$. We will denote by $[\gamma]$ the equivalence class of $\gamma$.

Notice that the above definition does not depend on the base point $o$ and the particular complete metric on $M$. Thus the number of ends of $M$ is a topological invariant of $M$.

The following lemma is a refined version of Proposition 2.2 in $[\mathrm{C}]$ and can be proved by the same argument.

Lemma 2.2. Let $M$ be as in the theorem. If $\left[\gamma_{1}\right]$ and $\left[\gamma_{2}\right]$ are two different ends of $M$, then for any $t_{1}, t_{2} \geq 0, d\left(\gamma_{1}\left(t_{1}\right), \gamma_{2}\left(t_{2}\right)\right) \geq t_{1}+t_{2}-2 a$.

In what follows, let $M^{n}$ be as in the theorem. By scaling, we may assume that $\operatorname{Ric}\left(M^{n}\right) \geq-(n-1)$.

Following Abresch and Gromoll in [AG], let $\phi(x)$ be the function defined on $B_{-1}(o, 1)-\{o\}$, the truncated unit ball in the hyperbolic space $\mathbf{H}^{n}$, with the following property:

$$
\begin{aligned}
\Delta \phi & =2(n-1), \\
\left.\phi\right|_{\partial B_{-1}(1)} & =0 .
\end{aligned}
$$


It is easy to see that $\phi(x)=G(d(o, x))$, where

$$
G(r)=2(n-1) \int_{r}^{1} \int_{t}^{1}\left(\frac{\sinh s}{\sinh t}\right)^{n-1} d s d t .
$$

Given a continuous function $u: M \rightarrow R$ and $x \in M$, a continuous function $u_{x}: M \rightarrow R$ is called an upper barrier of $u$ at $x$ if $u_{x}(x)=u(x)$ and $u \leq u_{x}$. The following lemma is a slight generalization of Theorem 2.1 in [AG].

Lemma 2.3. Let $M^{n}$ be a complete Riemannian manifold with Ricci curvature bounded from below by $-(n-1)$. Then there exist an $\varepsilon=\varepsilon(n)>0$ and $a$ $\delta=\delta(n)>0$ such that

$$
u(x)<2-2 \delta-4 \varepsilon
$$

for all $x \in S(o, 1-\delta)$ if $u: M^{n} \rightarrow \mathbf{R}$ is a continuous function which satisfies the following properties:

$$
\begin{gathered}
u(o)=0, \\
u \geq-2 \varepsilon, \\
\operatorname{dil}(u) \leq 2, \\
\Delta u \leq 2(n-1),
\end{gathered}
$$

where $\operatorname{dil}(u)=\sup _{x \neq y}|u(x)-u(y)| / d(x, y)$ and the last inequality is in the barrier sense, that is, for any $x \in M$ and $\alpha>0$, there is an upper barrier of $u$ at $x, u_{x, \alpha}$, such that $u_{x, \alpha}$ is smooth near $x$ and $\Delta u_{x, \alpha}(x) \leq 2(n-1)+\alpha$.

Proof. Consider $H(r)=2 r+G(r)$. Notice that $G(1)=0$ and $G^{\prime}(1)=0$. Hence $H(1)=2$ and $H^{\prime}(r)>0$ for $r$ close to 1 , and therefore there exists a $c$ such that $0<c<1$ and $H(c)<2$. Now choose $\delta=\delta(n)$ and $\varepsilon=\varepsilon(n)$ such that

$$
0<\delta<\frac{1}{2} \min \{2-H(c), 1-c\}
$$

and

$$
0<\varepsilon<\frac{1}{2} \min \{G(1-\delta), 2-H(c)-2 \delta\}
$$

Consider the function $v(y)=u(y)-G(d(x, y))$ on the annulus $B(x, 1) \backslash$ $B(x, c)$. The well-known Laplacian comparison theorem for distance functions (cf. $[\mathrm{EH}]$ ) implies that $\Delta v \leq 0$ (in the barrier sense). By the maximum principle [EH], $v$ achieves its minimum on the boundary of the annulus. Since $o$ is an interior point of the domain by (5) and $v(o)=u(o)-G(d(o, x))=-G(1-\delta)<$ $-2 \varepsilon$ by (6), there exists a point $z$ on the boundary of the domain such that $v(z)<-2 \varepsilon$. But on $S(x, 1), v=u-G(1)=u \geq-2 \varepsilon$ by (2). Hence $z \in S(x, c)$. Combining this with (3) and (6), we conclude that

$$
u(x) \leq u(z)+2 c=v(z)+H(c)<2-2 \delta-4 \varepsilon .
$$

This proves Lemma 2.3.

Remark 2.4. For a ray $\gamma$ in $M$, let $b_{\gamma}$ be the associated Busemann function, i.e.,

$$
b_{\gamma}(x)=\lim _{t \rightarrow \infty}(d(\gamma(t), x)-t)
$$

It is well known (e.g., see [EH]) that, in the barrier sense, $\Delta b_{\gamma} \leq n-1$. We are now in position to prove the theorem. 
Proof of the theorem. Let $M^{n}$ be as in the theorem with $\Lambda=1$. Let $\varepsilon=\varepsilon(n)$ be as in Lemma 2.3. We need to show that when $a \leq \varepsilon, M^{n}$ has at most two ends. Suppose not. Let $\left[\gamma_{1}\right],\left[\gamma_{2}\right]$, and $\left[\gamma_{3}\right]$ be three different ends. Consider $u:=b_{\gamma_{1}}+b_{\gamma_{2}}$. We claim that $u$ satisfies the conditions in Lemma 2.3. As a matter of fact, (1) and (3) are clear, (4) is by Remark 2.4, and (2) is a consequence of the triangle inequality and Lemma 2.2. From Lemma 2.3, we conclude that

$$
u\left(\gamma_{3}(1-\delta)\right)<2-2 \delta-4 \varepsilon \text {. }
$$

On the other hand, it follows from Lemma 2.2 that for any $t \geq 0$,

$$
u\left(\gamma_{3}(t)\right) \geq 2 t-4 a .
$$

In particular,

$$
u\left(\gamma_{3}(1-\delta)\right) \geq 2(1-\delta)-4 a \geq 2-2 \delta-4 \varepsilon
$$

This clearly contradicts (7) and hence completes the proof of the theorem.

\section{REFERENCES}

[A] U. Abresch, Lower curvature bounds, Toponogov's theorem and bounded topology, Ann. Sci. École Norm. Sup. (4) 18 (1985), 651-670.

[AG] U. Abresch and D. Gromoll, On complete manifolds with nonnegative Ricci curvature, J. Amer. Math. Soc. 3 (1990), 355-374.

[C] M. Cai, Ends of Riemannian manifolds with nonnegative Ricci curvature outside a compact set, Bull. Amer. Math. Soc. (N.S.) 24 (1991), 371-377.

[CG] J. Cheeger and D. Gromoll, The splitting theorem for manifolds of nonnegative Ricci curvature, J. Differential Geom. 6 (1971), 119-128.

[EH] J.-H. Eschenburg and E. Heintze, An elementary proof of the Cheeger-Gromoll splitting theorem, Ann. Global Anal. Geom. 2 (1984), 249-260.

[L] Z. Liu, Ball covering on manifolds with nonnegative Ricci curvature near infinity, Proc. Amer. Math. Soc. 115 (1992), 211-219.

[LT] P. Li and F. Tam, Harmonic functions and the structure of complete manifolds, preprint, 1990.

[SY] J. P. Sha and D. G. Yang, Positive Ricci curvature on the connected sums of $S^{n} \times S^{m}, \mathrm{~J}$. Differential Geom. 33 (1991), 127-137.

(M. Cai and T. H. Colding) Department of Mathematics, University of Pennsylvania, Philadelphia, Pennsylvania 19104

Current address, M. Cai: Department of Mathematics and Computer Science, University of Miami, Coral Gables, Florida 33124

E-mail address: mcai@math.miami.edu

Current address, T. H. Colding: Courant Institute, New York University, New York, New York 10012

(D. Yang) Department of Mathematics, Tulane University, New Orleans, Louisiana 70118 